\title{
Vertucci's Root Canal Configuration of 11376 Mandibular Anteriors and its Relationship with Distolingual Roots in Mandibular First Molars in A Cantonese Population: A Cone-Beam Computed Tomography Study
}

\section{Yeqing Yang}

Stomatological Hospital of Southern Medical University

\section{Chong Jiang}

Guangdong Academy of Medical Sciences, Guangdong Provincial People's Hospital

\section{Ming Chen}

Stomatological Hospital of Southern Medical University

Junkai Zeng

Southern Medical University

Buling Wu ( $\sim$ Mailbulingwu@smu.edu.cn )

Southern Medical University

\section{Research Article}

Keywords: Cone-beam computed tomography, Mandibular anteriors, Distolingual roots, Vertucci's root canal configuration

Posted Date: December 15th, 2021

DOI: https://doi.org/10.21203/rs.3.rs-1080802/v1

License: (a) (i) This work is licensed under a Creative Commons Attribution 4.0 International License. Read Full License 


\section{Abstract}

Background: Cone-beam computed tomography (CBCT) was used to study the root canal system of mandibular anteriors (MAs) in a Cantonese population and to evaluate the correlation between the complicated root canal configurations of mandibular lateral incisors (MLIs) and the presence of distolingual roots (DLRs) in mandibular first molars (MFMs).

Methods: A total of 11376 mandibular anterior teeth were scanned by CBCT. Those whose images met the inclusion criteria were first analysed according to Vertucci's root canal configuration and then grouped based on gender, age, and side, and their effects on root canal morphology were analysed. Finally, statistical analysis was used to evaluate the correlation between the complicated root canal configurations of MLIs and the existence of DLRs in MFMs. All statistical analyses were performed by using SPSS 21.0 software. Quantitative data are presented as the mean \pm standard deviation. Student's $t$ tests were used to calculate statistical significance. $\mathrm{P}<0.05$ was considered statistically significant.

Results: In MAs in the Cantonese population, all mandibular central incisors (MCls) and MLIs had one root, and $0.37 \%$ of mandibular canines (MCs) had two roots. The most common Vertucci's root canal configuration was Vertucci I followed by Vertucci III. A total of $30.91 \%$ of MLIs and approximately $8 \%$ of $\mathrm{MCl}$ and MCs have complicated root canal configurations. There were no significant differences in the prevalence of DLRs in MFMs and the incidence of complicated root canal configurations of MLIs between males and females or between the right and left teeth. However, a significant difference was found in different age groups of root canal configurations in MLIs. Moreover, significant ipsilateral and contralateral correlations between MFMs with DLRs and MLIs with complicated root canal configurations were observed on both sides.

Conclusion: There is a correlation between the presence of DLRs in MFMs and complicated root canal morphology in $\mathrm{MCls}$ in the Cantonese population.

\section{Background}

Successful root canal treatment is very challenging because of increased difficulties in cleaning, shaping, and filling the root canal system in a 3-dimensional manner[1-3]. A comprehensive understanding of the complexity of the root canal system is necessary for successful endodontic treatment. Considering that ethnic factors will affect root canal configuration, it is necessary to study different populations around the world[4].

Since 1990, cone-beam computed tomography (CBCT) has been used in the field of endodontics[5]. It provides a noninvasive technique to more accurately assess the root canal system and helps to perform detailed research on internal anatomy. Compared with the periapical, panoramic and micro CT techniques, it has the advantages of a small radiation dose, high image resolution, 3D reconstruction, and simple operation [6]. CBCT images can be analysed by computer and simultaneously displayed in the sagittal, coronal and axial planes, which is particularly suitable for oral clinical needs. 
Mandibular first molars (MFMs) are the first permanent molars to grow into the mouth. Because of pulpitis and periapical periodontitis, root canal treatment is often needed[7]. The root canal system of the MFM is one of the most complicated among human teeth. For example, the system is characterized by a c-shaped root canal[8], distolingual roots (DLRs)[9], middle mesial canals and the occurrence of fusion roots [10]. The DLR, also known as radix entomolaris, is one of the major variants in MFMs[11-14]. The complexity of the root and canal morphology of MFMs can be attributed to ethnicity, age, gender, and study design[15]. Even if the person has partial edentulism, the long-standing teeth in the mouth are still mandibular permanent incisors[16]. Many clinicians mistakenly believe that the anterior mandibular teeth all have single root canals. However, the root canal configurations of mandibular anterior teeth may vary greatly with ethnicity and gender. In particular, a mandibular anterior tooth may have additional canals and multiple canal configurations, which may have a key impact on endodontic treatments. Based on the population studied, CBCT studies have demonstrated a high prevalence of second canals in central and lateral incisors. Second canals were found in approximately $15 \%$ of the population in Iran and Turkey [17, 18]. The prevalence of two root canals in mandibular anterior teeth in Brazilians was approximately $20 \%$ for central and lateral incisors and $10 \%$ for canines. Another study in the Chinese population showed that the prevalence for central incisors was similar, but the prevalence for lateral incisors was higher (27\%)[19]. The Malay ethnic group showed more canal variations in mandibular incisors than the Chinese and Indian groups[20].

Although many authors have studied mandibular lateral incisors (MLIs) using CBCT, few studies have reported on the morphological classification in a Cantonese population. The presence of DLR is related to many morphologic features, such as crown dimension[21], interorifice distance of canals[12], and distance from buccal cortical bone[22]. However, in previous studies, a possible association between the prevalence of DLR in MFM and the morphological characteristics of other teeth has been investigated. The cooccurrence of DLRs in MFMs and the complicated root canal morphology in mandibular central incisors ( $\mathrm{MCls}$ ) are prominent among Taiwanese patients [23]. However, there is no research report among Cantonese. The main purpose of this study was to provide an in vivo assessment of the prevalence of the different Vertucci's root canal configurations in mandibular anterior teeth in a Cantonese population. $\mathrm{CBCT}$ images were used to determine the correlation between the root canal configurations of $\mathrm{MCls}$ and the appearance of DLR in MFMs.

\section{Methods}

\section{Patients}

CBCT images from 2356 Cantonese patients in this study were obtained between January 2018 and January 2019 using the CBCT imaging system and collected from the database of the Department of Oral Radiology, Stomatological Hospital, Southern Medical University, Guangzhou. All patients required radiographic examination of $\mathrm{CBCT}$ as part of their dental treatment. The images were taken as part of the routine examination, diagnosis, and treatment planning for patients who included those suffering facial trauma or maxillary sinusitis, who required oral surgery or orthodontic treatment or who needed implant 
treatment. This project and protocol were approved by the Medical Ethics Committee of Nanfang Hospital (NFEC-2020-106). CBCT images were obtained using a Planmeca Romexis3D CBCT scanner (Planmeca, Finland). Board-certified radiologists operated the X-ray tube at an accelerated potential with a field-ofview size (FOV) of $8 \times 8 \mathrm{~cm}$, a peak voltage of $84 \mathrm{kV}$, a beam current of $14 \mathrm{~mA}$ and an exposure time of $12 \mathrm{~s}$ for a full arch. The voxel size was $200 \mu \mathrm{m} \times 200 \mu \mathrm{m}$, and the minimum layer thickness was 0.15 $\mathrm{mm}$. A total of 1896 patients' images qualified for further analysis based on the following inclusion criteria:

1. The mandibular central incisors, mandibular lateral incisors, mandibular canines $₫$ permanent mandibular first molars were present bilaterally with complete root formation

2. Absence of root canal treatment

3. Complete root formation without evident resorption process

4. Absence of periapical disease

5. Absence of coronal or post and core restorations, which may obscure the imaging study

6. Good quality CBCT images that are clear and free of artefacts.

\section{Morphologic Analysis and Classification}

The image data were exported in DICOM format. To assess data reliability, all images were repositioned and examined in a dimly lit environment separately by two endodontists. Serial axial, coronal, and sagittal $\mathrm{CBCT}$ images were thoroughly examined from the pulp orifice to the apical foramen. In cases of disagreement, these two endodontists discussed the data until a consensus was reached. An oral radiologist provided guidance when necessary. The presence or absence of DLRs in MFMs was defined according to previous studies[23, 24] (Figure 1).

1. Non-DLR: the patient has MFMs without DLRs

2. Unilateral DLR (Uni-DLR): the patients has DLR only on the left or right MFM and no DLR on the other side

3. Bilateral DLR (Bil-DLR): the patients has DLRs on both left and right MFMs

Based on previous studies on the MLIs, we classified them into the following categories [23](Figure 1) $₫$

1. Single: a single canal, with only one canal from the pulp orifice to the apical in MLIs.

2. Complicated: complicated canal『with more than one canal from the pulp orifice to the apical in MLIs.

The single or complicated canal in MLIs was further categorized as follows: 
1. Unilateral: the patients had a"single canal"or"complicated canal" in either the left or right MLIs

2. Bilateral: the patients had a"single"or "complicated canal"in MLIs bilaterally

In CBCT images, the root canal system of MLIs was classified based on classic Vertucci classification[7] (Figure 2).

The contralateral teeth were compared for symmetry of the root canal configuration. The influence of gender on the canal configuration was also analyzed.

1. Type I (1-1): Pulp chamber bottom have one root canal orifices, and always have one independent root canal

2. Type II (2-1): The pulp chamber bottom has two root canal orifices. Then, these orifices merge into one canal at a certain position of the root canal to yield one apical foramens.

3. Type III (1-2-1): The bottom of the pulp chamber has one root canal orifices that branch into two separate root canals. These canals finally merge into one root canal.

4. Type IV (2-2): Two separate canals extend from the pulp chamber to the apical.

5. Type V (1-2): The pulp chamber has one canal and divides short of the apical into two separate canals, with separate apical foramens.

6. Type VI (2-1-2): The pulp chamber has two separate canals, join at the midpoint, and then divide again into two with two separate apical foramens.

7. Type VII (1-2-1-2):At the bottom of the pulp chamber, there is one root canal orifice that branch into two root canals, fuse into one root canal, and finally emerge through two different apical holes out of the root canal system

8. Type VIII (3-3): Pulp chamber bottom have three root canal orifices, and always have three independent root canals, and finally there are three different apical foramens.

9. *Type IX (2-1-2-1):Two root canals are located at the beginning of the pulp chamber and subsequently merges into one root canal, then branches into two independent root canals. Finally, the same apical foramen is formed.

10. *Type X (1-2-3-1): Only one root canal orifice is located at the bottom of the pulp chamber. The orifice branches into two independent root canals, subsequently divides into three independent root canals, and finally merges into the same root canal at the apex part of the root canal.

The contralateral teeth were compared for symmetry of the root canal configuration. The influence of gender on the canal configuration was also analyzed. 


\section{Statistical analysis}

Statistical analysis was performed using SPSS(Version21.0; SPSS, Inc, Chicago, IL), a statistical software package for Windows. The measurement data were expressed as the mean standard deviation or percentages as appropriate for each measurement calculated at the individual and tooth levels. The chisquare test was used to analyze differences among categorical variables, such as age(age $<45 \mathrm{vs}$. age $\geq$ 45 years) $\bigotimes$ gender(male vs. female), and side(left vs. right) and DLR group(Non-DLR, Uni-DLR, or Bi-DLR). The mean is compared using $t$ tests with significance set at $P<0.05$.

\section{Results}

Of the 2356 patient CBCT images initially scanned, 1896 patients (3792 MLIs and 3792 MFMs) were eligible for further analysis. Of these subjects, 1016 (53.59\%) were females and 880 (46.41\%) were males, with an average age of $41.22 \pm 12.23$ years and $40.56 \pm 14.56$ years, respectively (Table 1 ).

Table 1

Characteristics of the Study Cantonese Population of the teeth

\begin{tabular}{|lllllll|}
\hline & Number of patients & Age(years) & & & \multicolumn{2}{c|}{ Number of teeth $(\mathbf{n})$} \\
\hline & $\mathrm{n}(\%)$ & Mean \pm SD & Max & Min & MLIs & MFMs \\
\hline Female & $1016(53.59 \%)$ & $41.22 \pm 12.23$ & 72 & 18 & 2032 & 2032 \\
\hline Male & $880(46.41 \%)$ & $40.56 \pm 14.56$ & 70 & 18 & 1760 & 1760 \\
\hline Total & $1896(100.00 \%)$ & $40.86 \pm 13.77$ & 72 & 18 & 3792 & 3792 \\
\hline
\end{tabular}

A total of $11376 \mathrm{MCls}$, MLIs and MCs were assessed. Based on Vertucci's root canal configuration (1984), the distribution of teeth is shown in Table 2. Among them, the most common root canal type for MAs was Vertucci type I at $84.65 \%$ (9630/11376), followed by Vertucci type III at 14.39\% (1637/11376): $7.60 \%$ (288/3792) of MCls, $7.54 \%$ (286/3792) of MCs, and $30.91 \%$ (1172/3792) of MLIs were complicated root canal configurations. In addition, we also discovered two new root canal types of the MCs, which were type IX (2-1-2-1) and type X (1-2-3-1) (Table 2). All MCls and MLIs had only one root, and $0.37 \%(14 / 3792)$ of MCs had two roots (Table 3 ). 
Table 2

Incidence of different root canal morphologies of mandibular anterior

\begin{tabular}{|c|c|c|c|c|c|c|}
\hline Vertucci's classification & 33 & 32 & 31 & 41 & 42 & 43 \\
\hline \multirow[t]{2}{*}{ Type I(1-1) } & 1750 & 1312 & 1754 & 1750 & 1308 & 1756 \\
\hline & $(92.30 \%)$ & $(69.20 \%)$ & $(92.51 \%)$ & $(92.30 \%)$ & $(68.99 \%)$ & $(92.62 \%)$ \\
\hline \multirow[t]{2}{*}{ Type II(2-1) } & 2 & 6 & 0 & 0 & 6 & 0 \\
\hline & $(0.11 \%)$ & $(0.32 \%)$ & $(0.00 \%)$ & $(0.00 \%)$ & $(0.32 \%)$ & $(0.00 \%)$ \\
\hline \multirow[t]{2}{*}{ Type III(1-2-1) } & 124 & 554 & 136 & 140 & 554 & 129 \\
\hline & $(6.54 \%)$ & $(29.22 \%)$ & $(7.17 \%)$ & $(7.38 \%)$ & $(29.22 \%)$ & $(6.80 \%)$ \\
\hline \multirow[t]{2}{*}{ Type IV(2-2) } & 0 & 0 & 0 & 0 & 2 & 0 \\
\hline & $(0.00 \%)$ & $(0.00 \%)$ & $(0.00 \%)$ & $(0.00 \%)$ & $(0.11 \%)$ & $(0.00 \%)$ \\
\hline \multirow[t]{2}{*}{ Type V(1-2) } & 16 & 24 & 6 & 6 & 26 & 8 \\
\hline & $(0.84 \%)$ & $(1.27 \%)$ & $(0.32 \%)$ & $(0.32 \%)$ & $(1.37 \%)$ & $(0.42 \%)$ \\
\hline \multirow[t]{2}{*}{ Type VI(2-1-2) } & 0 & 0 & 0 & 0 & 0 & 0 \\
\hline & $(0.00 \%)$ & $(0.00 \%)$ & $(0.00 \%)$ & $(0.00 \%)$ & $(0.00 \%)$ & $(0.00 \%)$ \\
\hline \multirow[t]{2}{*}{ Type VII(1-2-1-2) } & 2 & 0 & 0 & 0 & 0 & 0 \\
\hline & $(0.11 \%)$ & $(0.00 \%)$ & $(0.00 \%)$ & $(0.00 \%)$ & $(0.00 \%)$ & $(0.00 \%)$ \\
\hline \multirow[t]{2}{*}{ Type VIII (3-3) } & 0 & 0 & 0 & 0 & 0 & 0 \\
\hline & $(0.00 \%)$ & $(0.00 \%)$ & $(0.00 \%)$ & $(0.00 \%)$ & $(0.00 \%)$ & $(0.00 \%)$ \\
\hline \multirow[t]{2}{*}{ *Type IX (2-1-2-1) } & 2 & 0 & 0 & 0 & 0 & 2 \\
\hline & $(0.11 \%)$ & $(0.00 \%)$ & $(0.00 \%)$ & $(0.00 \%)$ & $(0.00 \%)$ & $(0.11 \%)$ \\
\hline \multirow[t]{2}{*}{ *Type X (1-2-3-1) } & 0 & 0 & 0 & 0 & 0 & 1 \\
\hline & $(0.00 \%)$ & $(0.00 \%)$ & $(0.00 \%)$ & $(0.00 \%)$ & $(0.00 \%)$ & $(0.05 \%)$ \\
\hline Total & 1896 & 1896 & 1896 & 1896 & 1896 & 1896 \\
\hline
\end{tabular}


Table 3

Incidence of the number of roots of mandibular anterior

\begin{tabular}{|lllllll|}
\hline Number of roots & $\mathbf{3 3}$ & $\mathbf{3 2}$ & $\mathbf{3 1}$ & $\mathbf{4 1}$ & $\mathbf{4 2}$ & $\mathbf{4 3}$ \\
\hline 1 & 1886 & 1896 & 1896 & 1896 & 1896 & 1892 \\
& $(99.47 \%)$ & $(100.00 \%)$ & $(100.00 \%)$ & $(100.00 \%)$ & $(100.00 \%)$ & $(99.78 \%)$ \\
\hline 2 & $10(0.52 \%)$ & $0(0.00 \%)$ & $0(0.00 \%)$ & $0(0.00 \%)$ & $0(0.00 \%)$ & $4(0.21 \%)$ \\
\hline
\end{tabular}

At the patient-level analysis, among 1016 females, bilateral complicated (Bil-Comp) root canals and bilateral single (Bil-Single) root canals were found in $65.35 \%$ (664/1016) and $24.61 \%$ (250/1016) of teeth, and $10.04 \%(102 / 1016)$ of the patients had inconsistent root canal configurations. Among 880 males, BilComp root canals and Bil-Single root canals were found in $62.50 \%(550 / 880)$ and $27.27 \%(240 / 880)$ of teeth, and $10.23 \%(90 / 880)$ of patients had inconsistent root canal configurations. The prevalence of bilateral (Bil-DLR) and unilateral (Uni-DLR) DLRs in MFMs was 13.00\% (132/1016) and 18.90\% (192/1016), respectively, in the female group. In the male group, the prevalence of Bil-DLR and Uni-DLR in MFMs was $15.68 \%$ (138/880) and 17.73\% (156/880), respectively (Figure 3$)$. There were no significant differences in the prevalence of DLRs in MFMs $(P=0.13)$ or the incidence of complicated root canal configurations of MLIs $(P=0.06)$ between males and females (Table 4$)$.

Table 4

Characteristics of the study population examined teeth by genders, age groups, and sides.

\begin{tabular}{|c|c|c|c|c|c|c|}
\hline & MLI & & & MFM & & \\
\hline & Single & Complicated & $P$ & Without DLR & With DLR & $P$ \\
\hline Total & 2620 & 1172 & & 2904 & 888 & \\
\hline \multicolumn{7}{|l|}{ Sex } \\
\hline Female & 1430 & 602 & \multirow[t]{2}{*}{$P=0.06$} & 1576 & 456 & \multirow[t]{2}{*}{$P=0.13$} \\
\hline Male & 1190 & 570 & & 1328 & 432 & \\
\hline \multicolumn{7}{|l|}{ Side } \\
\hline Left & 1312 & 584 & \multirow[t]{2}{*}{$P=0.89$} & 1475 & 421 & \multirow[t]{2}{*}{$P=0.07$} \\
\hline Right & 1308 & 588 & & 1429 & 467 & \\
\hline \multicolumn{7}{|l|}{ Age } \\
\hline$<45$ & 1762 & 996 & \multirow[t]{2}{*}{$P<0.0001$} & 2124 & 634 & \multirow[t]{2}{*}{$P=0.31$} \\
\hline$\geq 45$ & 858 & 176 & & 780 & 254 & \\
\hline
\end{tabular}

In addition, there were no significant differences in the prevalence of DLRs in MFMs $(P=0.07)$ or the incidence of complicated root canal configurations in MLIs $(P=0.89)$ between the left and right sides. We 
came to this result by using statistical methods (Table 4). In subjects with complicated MLIs, $30.80 \%$ (584/1896) were found in the left group and 31.01\% (588/1896) in the left group; moreover, of the DLRs in MFMs, $22.20 \%$ (421/1896) were found in the left group and 24.63\% (467/1896) were found in the right group (Figure 3).

There were significant differences in the prevalence of complicated root canal configurations in MLIs between age groups (age $<45$ years vs. age $\geq 45$ years, $P<0.0001$ ) (Table 4 ). The prevalence of Bil-DLR and Uni-DLR in MFMs was 19.07\% (263/1379) and 7.83\% (108/1379), respectively, in the age< 45 years group. In the age $\geq 45$ years group, the prevalence of Bil-DLR and Uni-DLR in MFMs was $19.92 \%(103 / 517)$ and $9.28 \%$ (156/517), respectively (Figure 3). There were no significant differences in the prevalence of DLRs in MFMs $(P=0.13)$ or the incidence of complicated root canal configurations of MLIs $(P=0.06)$ between males and females (Table 4).

Among the bilateral MFMs, 83.97\% (1592/1896) either both had or both did not have DLRs. Among the bilateral MLIs, $85.97 \%$ (1630/1896) had complicated root canal configurations on either both sides or neither side. These results reflect the high symmetry of the root canal morphology across bilateral teeth in the Cantonese population. In addition, regardless of whether the ipsilateral side or the contralateral side was examined, there was a correlation between the existence of DLR of the MFM and the complicated root canal configurations of the MLI (Table 5).

Table 5

Analysis of the Correlation of MFMs with DLRs and Root Canal Configurations of MLIs Ipsilaterally and Contralaterally

\begin{tabular}{|c|c|c|c|c|}
\hline & \multicolumn{4}{|l|}{ MFM, Right } \\
\hline & Without DLR & With DLR & Total & $P$ \\
\hline MFM, Left & & & & $<0.0001$ \\
\hline Without DLR & 1300 & 175 & 1475 & \\
\hline With DLR & 129 & 292 & 421 & \\
\hline Total & 1429 & 467 & 1896 & \\
\hline
\end{tabular}

\begin{tabular}{|c|c|c|c|c|}
\hline & \multicolumn{4}{|c|}{ MLI, Right } \\
\hline & Single & Complicated & Total & $P$ \\
\hline MLI, Left & & & & $<0.0001$ \\
\hline Single & 1177 & 135 & 1312 & \\
\hline Complicated & 131 & 453 & 584 & \\
\hline Total & 1308 & 588 & 1896 & \\
\hline
\end{tabular}




\begin{tabular}{|c|c|c|c|c|c|c|c|c|}
\hline & \multicolumn{4}{|c|}{ MLI, Left } & \multicolumn{4}{|c|}{ MLI, Right } \\
\hline & Single & Complicated & Total & $P$ & Single & Complicated & Total & $P$ \\
\hline \multicolumn{9}{|l|}{$\begin{array}{l}\text { MFM, } \\
\text { Left }\end{array}$} \\
\hline $\begin{array}{l}\text { Without } \\
\text { DLR }\end{array}$ & 1050 & 425 & 1475 & $P<0.0005$ & 1055 & 420 & 1475 & $P<0.0001$ \\
\hline $\begin{array}{l}\text { With } \\
\text { DLR }\end{array}$ & 262 & 159 & 421 & & 253 & 168 & 421 & \\
\hline Total & 1312 & 584 & 1896 & & 1308 & 588 & 1896 & \\
\hline \multicolumn{9}{|l|}{$\begin{array}{l}\text { MFM, } \\
\text { Right }\end{array}$} \\
\hline $\begin{array}{l}\text { Without } \\
\text { DLR }\end{array}$ & 1017 & 412 & 1429 & $P<0.0015$ & 1011 & 418 & 1429 & $P<0.004$ \\
\hline $\begin{array}{l}\text { With } \\
\text { DLR }\end{array}$ & 295 & 172 & 467 & & 297 & 170 & 467 & \\
\hline Total & 1312 & 584 & 1896 & & 1308 & 588 & 1896 & \\
\hline
\end{tabular}

\section{Discussion}

Carabelli first referred to DLR in 1884, often describing it as the presence of an additional lingual root and the major anatomical variation in MFM $[11-14,21,25,26]$. Previous studies have reported that the presence of this dental trait has been thought to be a genetically determined racial trait. In whites and blacks, the frequency of DLR is less than $5 \%$, while in populations with ethnic Mongolian characteristics (for example, Chinese, Japanese, Korean, and Taiwanese), the frequency of DLR ranges from 5-30\%[2729].

The complex anatomical variation in MCls makes it one of the most difficult teeth for root canal therapy[30, 31]. In this study, we evaluated the frequency of the Vertucci root canal configuration and the gender influence on its distribution in 11376 mandibular anterior teeth in a Cantonese subpopulation. This is a large sample size study. More than 3792 teeth of each type ( $\mathrm{MCl}, \mathrm{MLI}$ or $\mathrm{MC}$ ) were evaluated. Finally, we evaluated the correlation between the root canal configuration of $\mathrm{MLI}$ and the appearance of DLR in MFM.

This study found that all MCls and MLIs had single roots, which was consistent with previous studies conducted in Brazil[32], China[19] and Iran[33]. However, some studies have found that MLIs with two roots has been found in Turkish[18] and Chinese[34] populations, but the prevalence is low $(0.1 \%$ and $0.3 \%$, respectively). In addition, two root MCs were also observed in several other countries[18, 19, 33, 34], but the prevalence was low (0.8-4.7\%). The results of this study are lower than those reported in the literature, which may be due to ethnic differences. In our study, the presence of MFMs with DLR was 
significantly correlated with the complicated root canal configuration in PMCls. In other words, a significant ipsalateral correlation between MFMs with DLRs and PMLI with complicated root canal configuration was observed on both sides (left $(P<0.0005)$ and right $(P<0.0001)$ ). At the same time, correlations between MFM with DLR and MLI with the complicated root canal configuration were also observed on both sides (left $(P<0.0015)$ and right $(P<0.004))$. These results were different from those reported by Wu et al. in Taiwanese individuals [23], which may be caused by different regions and sample sizes.

Our research results on the morphology of mandibular incisors and the correlation between complicated root canal configurations of different teeth provide necessary knowledge to clinicians: in particular, the bilateral symmetry rate of DLR in MFMs, high levels of complicated canal configurations in MLIs, and a correlation between the two. Therefore, it is helpful for clinicians to treat teeth in a symmetrical manner.

Knowledge of root canal anatomy is essential for successful root canal treatment. Endodontic treatment of mandibular anterior teeth and mandibular first molar treatments can be challenging in the presence of additional root canals, which may not be recognized by periapical radiographs. Previous literature has reported a positive correlation between missing root canals and persistent apical periodontitis [2, 3, 35]. CBCT may be considered if the clinician suspects the presence of multiple root canals. The accuracy of CBCT is much higher than that of periapical radiographs[36, 37]. Therefore, clinicians should be aware that if a patient has complicated canal configurations in MLIs, there may be DLR in the MFMs and complicated anatomical structures in other teeth. However, due to the lack of research data, further studies are needed to investigate the relationships with the other teeth.

\section{Conclusion}

Among the MAs in the Cantonese population, the presence of complicated root canal configurations in MCls and MCs were $8 \%$ and MLIs were $30 \%$. More than $80 \%$ of the MAs and the contralateral teeth have symmetrical root canals. At the same time, this study showed that when there were DLRs in MFMs in the Cantonese population, the possibility of complicated root canal configuration in MCls was higher. Clinicians must not only be aware of the changes in the number of roots and root canal configurations but also be aware that the anatomical differences between teeth may vary and be correlated.

\section{Abbreviations}

CBCT: Cone-beam computed tomography; MA: mandibular anterior; MLI: mandibular lateral incisor; DLR: distolingual root; MFM: mandibular first molar; $\mathrm{MCl}$ : mandibular central incisors; MC: mandibular canine; Bil-Comp; bilateral complicated; Bil-Single; bilateral single; Bil-DLR: bilateral DLR; Uni-DLR; unilateral DLR

\section{Declarations}




\section{Ethics approval and consent to participate}

This study was approved by the Medical Ethics Committee of Nanfang Hospital (NFEC-2020-106).

\section{Consent to publication}

Not applicable.

\section{Availability of data and materials}

The datasets used and/or analysed during the current study are available from the corresponding author on reasonable request.

\section{Competing interests}

The authors have stated explicitly that there is no conflict of interests in connection with this article.

\section{Funding}

This work was supported by the General Program of National Natural Scientific Foundation of China (No.81870755)

\section{Author Contributions}

Yeqing Yang and Chong Jiang wrote the main manuscript text, collected and analysed data and prepared Tables 1-2, authors contribute equally to this article. Ming Chen collected data and prepared figures 1-3. Junkai Zeng prepared Tables 3-5. Buling Wu designed the study, performed critical revisions and analysed data. All authors reviewed the manuscript.

\section{Acknowledgements}

Not applicable.

\section{References}

1. Ahmed HMA, Versiani MA, De-Deus G, Dummer PMH. A new system for classifying root and root canal morphology. Int Endod J. 2017;50:761-70. 
2. Costa FFNP, Pacheco-Yanes J, Siqueira JF, Oliveira ACS, Gazzaneo I, Amorim CA, et al. Association between missed canals and apical periodontitis. Int Endod J. 2019;52:400-6.

3. Baruwa AO, Martins JNR, Meirinhos J, Pereira B, Gouveia J, Quaresma SA, et al. The Influence of Missed Canals on the Prevalence of Periapical Lesions in Endodontically Treated Teeth: A Crosssectional Study. Journal of Endodontics. 2020;46:34-39.e1.

4. Martins JNR, Marques D, Silva EJNL, Caramês J, Versiani MA. Prevalence Studies on Root Canal Anatomy Using Cone-beam Computed Tomographic Imaging: A Systematic Review. Journal of Endodontics. 2019;45:372-386.e4.

5. Patel S, Kanagasingam S, Mannocci F. Cone Beam Computed Tomography (CBCT) in Endodontics. Dental Update. 2010;37:373-9.

6. Kucher M, Dannemann M, Modler N, Hannig C, Weber M-T. An Automated Measurement Method for the Endodontic Working Width of Lower Molars by Means of Parametric Models Using Cone-beam Computed Tomographcy and Micro-Computed Tomography. Journal of Endodontics. 2021;:S0099239921005665.

7. Vertucci FJ. Root canal anatomy of the human permanent teeth. Oral Surgery, Oral Medicine, Oral Pathology. 1984;58:589-99.

8. Shemesh A, Levin A, Katzenell V, Itzhak JB, Levinson O, Avraham Z, et al. C-shaped canalsprevalence and root canal configuration by cone beam computed tomography evaluation in first and second mandibular molars-a cross-sectional study. Clin Oral Invest. 2017;21:2039-44.

9. Zhang X, Xu N, Wang H, Yu Q. A Cone-beam Computed Tomographic Study of Apical Surgery-related Morphological Characteristics of the Distolingual Root in 3-rooted Mandibular First Molars in a Chinese Population. Journal of Endodontics. 2017;43:2020-4.

10. de Pablo ÓV, Estevez R, Péix Sánchez M, Heilborn C, Cohenca N. Root Anatomy and Canal Configuration of the Permanent Mandibular First Molar: A Systematic Review. Journal of Endodontics. 2010;36:1919-31.

11. Zhang M, Xie J, Wang Y, Feng Y. Mandibular first premolar with five root canals: a case report. BMC Oral Health. 2020;20:253.

12. Tu M-G, Huang H-L, Hsue S-S, Hsu J-T, Chen S-Y, Jou M-J, et al. Detection of Permanent Three-rooted Mandibular First Molars by Cone-Beam Computed Tomography Imaging in Taiwanese Individuals. Journal of Endodontics. 2009;35:503-7.

13. Huang R-Y, Cheng W-C, Chen C-J, Lin C-D, Lai T-M, Shen E-C, et al. Three-dimensional analysis of the root morphology of mandibular first molars with distolingual roots: Distolingual root morphology. International Endodontic Journal. 2010;43:478-84.

14. Huang R-Y, Lin C-D, Lee M-S, Yeh C-L, Shen E-C, Chiang C-Y, et al. Mandibular Disto-Lingual Root: A Consideration in Periodontal Therapy. Journal of Periodontology. 2007;78:1485-90.

15. Ho DKL, Wong JHL, Pelekos G, Deng K, Tonetti MS. Prevalence and morphological characteristics of disto-lingual roots in mandibular first molars: a cone beam CT study with diagnostic and therapeutic implications. Clin Oral Invest. 2021;25:4023-30. 
16. Aung NM, Myint KK. Evidence of Second Canal between Permanent Mandibular Central and Lateral Incisors in China; a Systematic Review on CBCT Studies. International Journal of Dentistry. 2020;2020:1-7.

17. Haghanifar S, Moudi E, Bijani A, Ghanbarabadi MK. Morphologic assessment of mandibular anterior teeth root canal using CBCT. ama. 2018;46:85.

18. Kayaoglu G, Peker I, Gumusok M, Sarikir C, Kayadugun A, Ucok O. Root and canal symmetry in the mandibular anterior teeth of patients attending a dental clinic: CBCT study. Braz oral res. 2015;29:17.

19. Han T, Ma Y, Yang L, Chen X, Zhang X, Wang Y. A Study of the Root Canal Morphology of Mandibular Anterior Teeth Using Cone-beam Computed Tomography in a Chinese Subpopulation. Journal of Endodontics. 2014;40:1309-14.

20. Karobari MI, Noorani TY, Halim MS, Ahmed HMA. Root and canal morphology of the anterior permanent dentition in Malaysian population using two classification systems: A CBCT clinical study. Aust Endod J. 2021;47:202-16.

21. Kim KR, Song JS, Kim S-O, Kim SH, Park W, Son H-K. Morphological changes in the crown of mandibular molars with an additional distolingual root. Archives of Oral Biology. 2013;58:248-53.

22. Kim S-Y, Yang S-E. Cone-Beam Computed Tomography Study of Incidence of Distolingual Root and Distance from Distolingual Canal to Buccal Cortical Bone of Mandibular First Molars in a Korean Population. Journal of Endodontics. 2012;38:301-4.

23. Wu Y-C, Cathy Tsai Y-W, Cheng W-C, Weng P-W, Su C-C, Chiang H-S, et al. Relationship of the Incidence of C-shaped Root Canal Configurations of Mandibular First Premolars with Distolingual Roots in Mandibular First Molars in a Taiwanese Population: A Cone-beam Computed Tomographic Study. Journal of Endodontics. 2018;44:1492-1499.e1.

24. Wu Y-C, Cheng W-C, Weng P-W, Chung M-P, Su C-C, Chiang H-S, et al. The Presence of Distolingual Root in Mandibular First Molars Is Correlated with Complicated Root Canal Morphology of Mandibular Central Incisors: A Cone-beam Computed Tomographic Study in a Taiwanese Population. J Endod. 2018;44:711-716.e1.

25. Chen G, Yao H, Tong C. Investigation of the root canal configuration of mandibular first molars in a Taiwan Chinese population. International Endodontic Journal. 2009;42:1044-9.

26. Chen Y-C, Lee Y-Y, Pai S-F, Yang S-F. The Morphologic Characteristics of the Distolingual Roots of Mandibular First Molars in a Taiwanese Population. Journal of Endodontics. 2009;35:643-5.

27. Rodríguez-Niklitschek CA, Oporto GH, Garay I, Salazar LA. Clinical, imaging and genetic analysis of double bilateral radix entomolaris. Folia Morphol (Warsz). 2015;74:127-32.

28. Schäfer E, Breuer D, Janzen S. The Prevalence of Three-rooted Mandibular Permanent First Molars in a German Population. Journal of Endodontics. 2009;35:202-5.

29. Sperber, Moreau. Study of the number of roots and canals in Senegalese first permanent mandibular molars. International Endodontic Journal. 1998;31:117-22. 
30. Mitta S. Endodontic Management of a Mandibular Central Incisor with Type IV Canal Pattern: A Case Report. JCDR. 2014. https://doi.org/10.7860/JCDR/2014/6896.4180.

31. Tabassum S, Khan FR. Failure of endodontic treatment: The usual suspects. Eur J Dent. 2016;10:144-7.

32. Estrela C, Bueno MR, Couto GS, Rabelo LEG, Alencar AHG, Silva RG, et al. Study of Root Canal Anatomy in Human Permanent Teeth in A Subpopulation of Brazil's Center Region Using Cone-Beam Computed Tomography - Part 1. Braz Dent J. 2015;26:530-6.

33. Aminsobhani M, Sadegh M, Meraji N, Razmi H, Kharazifard MJ. Evaluation of the root and canal morphology of mandibular permanent anterior teeth in an Iranian population by cone-beam computed tomography. J Dent (Tehran). 2013;10:358-66.

34. Zhou Z, Yang Z, Lu K, Wang F, Li Y. Cone-beam computed tomography study of the root and canal morphology of mandibular permanent anterior teeth in a Chongqing population [Corrigendum]. TCRM. 2016;:387.

35. Karabucak B, Bunes A, Chehoud C, Kohli MR, Setzer F. Prevalence of Apical Periodontitis in Endodontically Treated Premolars and Molars with Untreated Canal: A Cone-beam Computed Tomography Study. Journal of Endodontics. 2016;42:538-41.

36. Gomez F, Brea G, Gomez-Sosa JF. Root canal morphology and variations in mandibular second molars: an in vivo cone-beam computed tomography analysis. BMC Oral Health. 2021;21:424.

37. Yang Y, Wu B, Zeng J, Chen M. Classification and morphology of middle mesial canals of mandibular first molars in a southern Chinese subpopulation: a cone-beam computed tomographic study. BMC Oral Health. 2020;20:358.

\section{Figures}
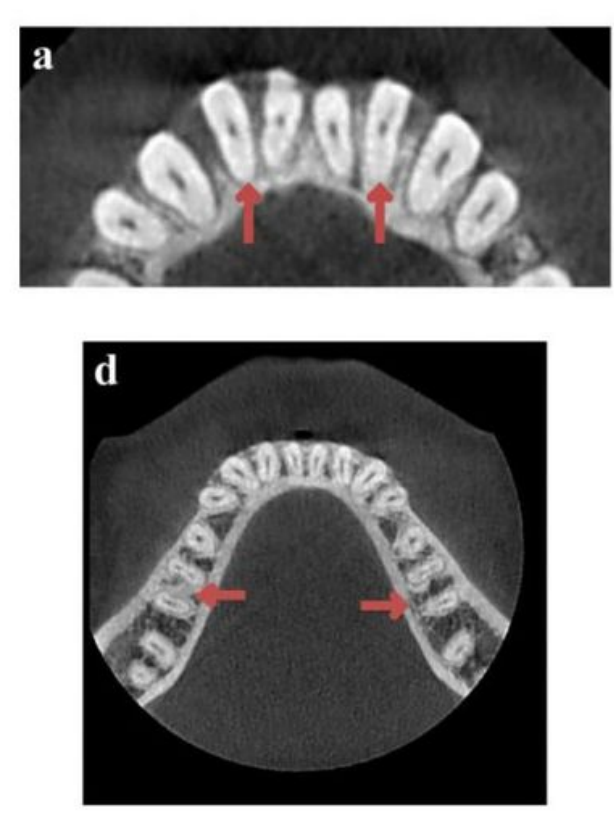
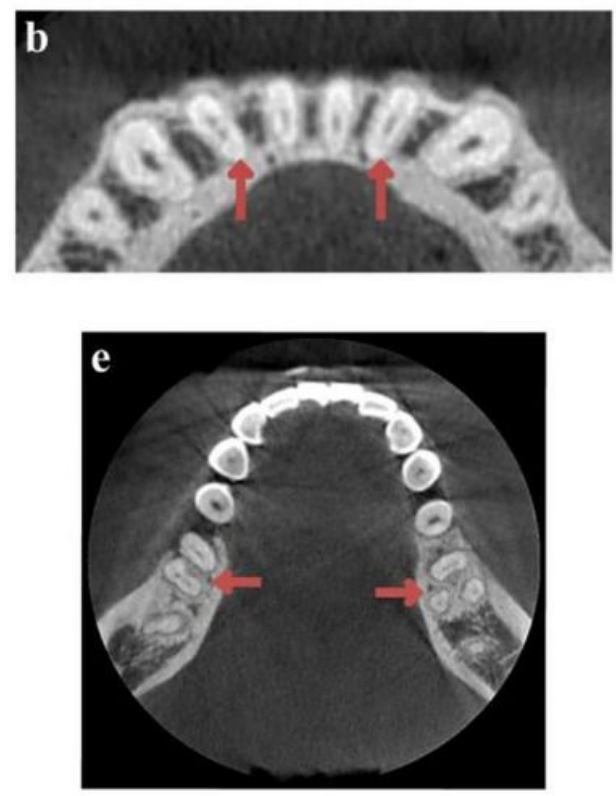
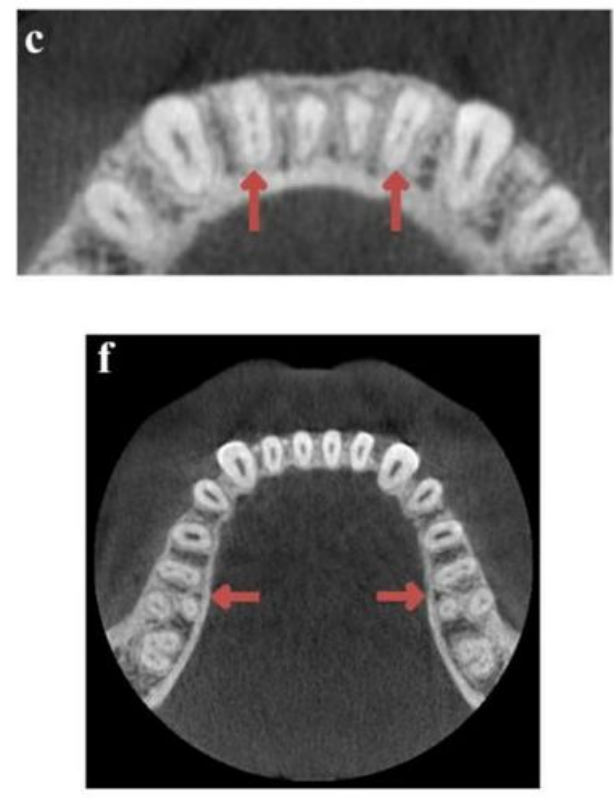
Figure 1

Representative images of root canal configurations of MLIs and the presence or absence of DLRs in MFMs. a Both MLIs were single root canal. b Right side or left side was single root canal. c Both MLIs were complicated root canals. d DLRs were found in both MFMs (Bi-DLR). e DLR was found in right side or left side MFM (Uni-DLR). $f$ No DLR was found in both MFMs (Non-DLR).

Coronal

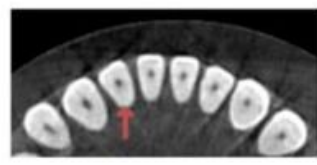

Type II(2-1)

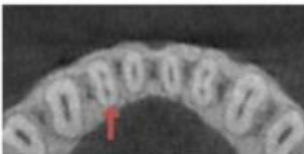

Type III(1-2-1)

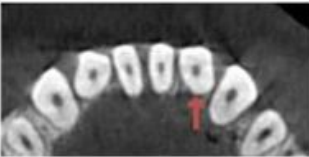

Type IV(2-2)

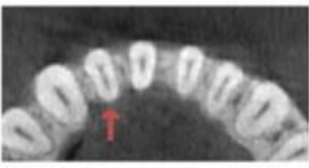

Type V(1-2)

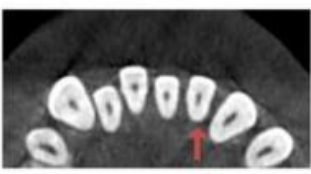

Type VII (1-2-1-2)
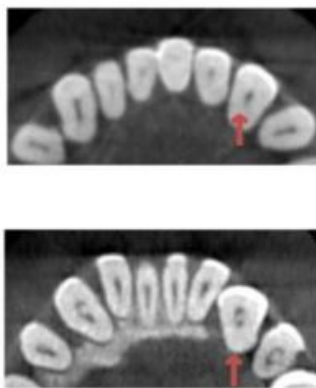

*Type IX (2-1-2-1)

*Type X(1-2-3-1)
Middle
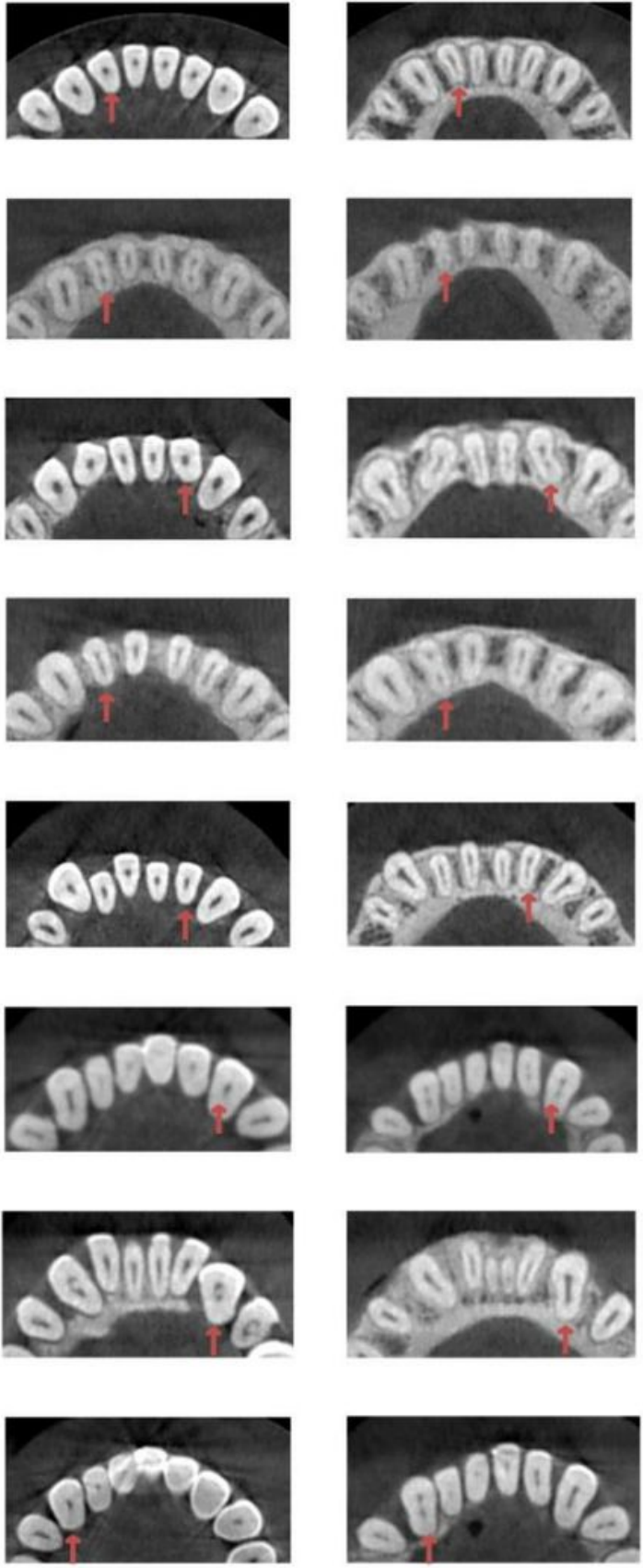

Apical
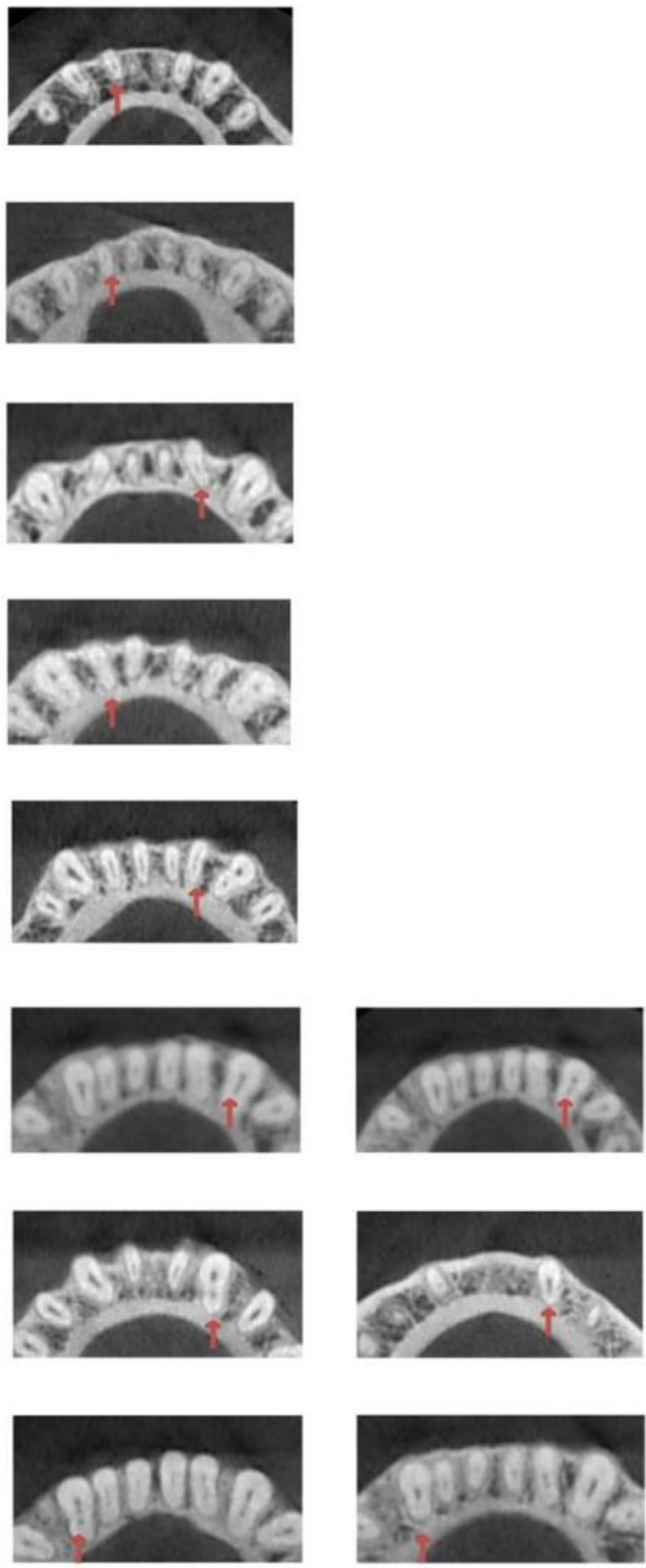

Figure 2 
The root canal configuration of MAs were classified according to the Vertucci classification. * We found that there were two classifications have not been reported by using CBCT

a

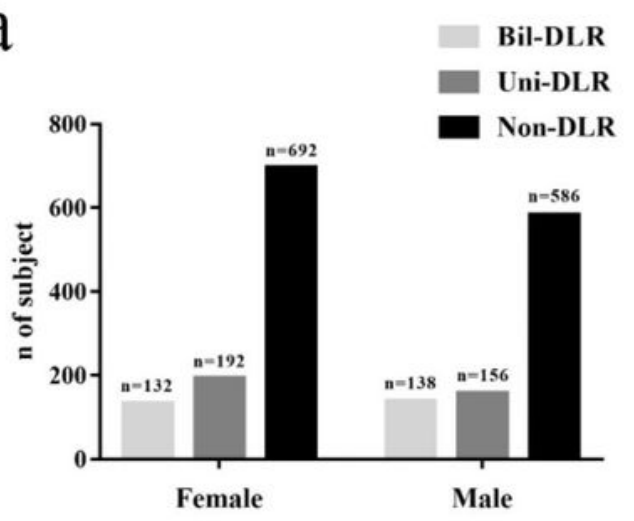

C
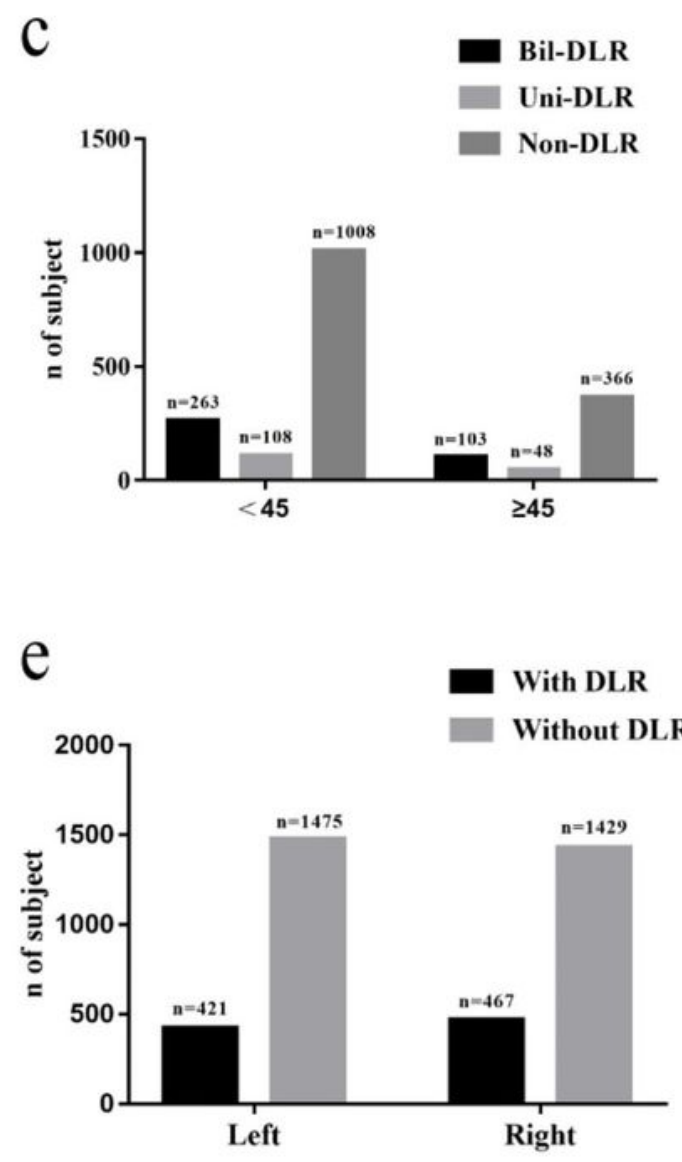

$\mathrm{b}$

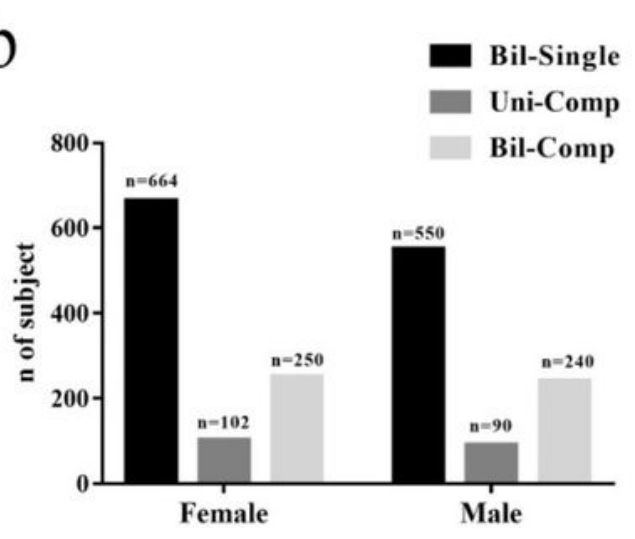

d

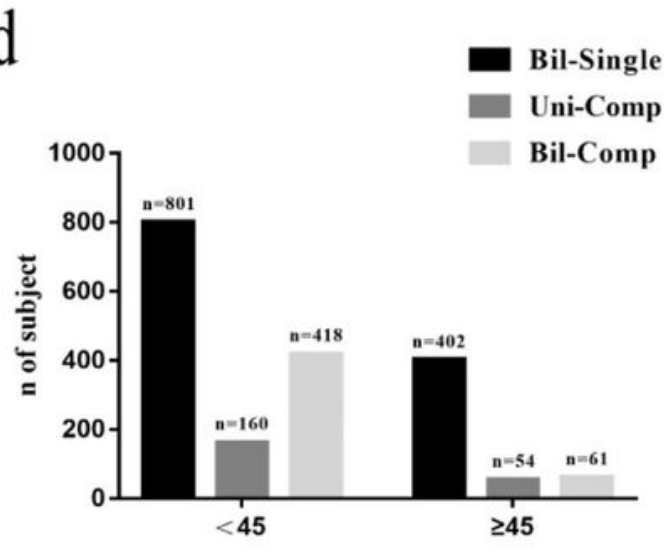

$\mathrm{f}$

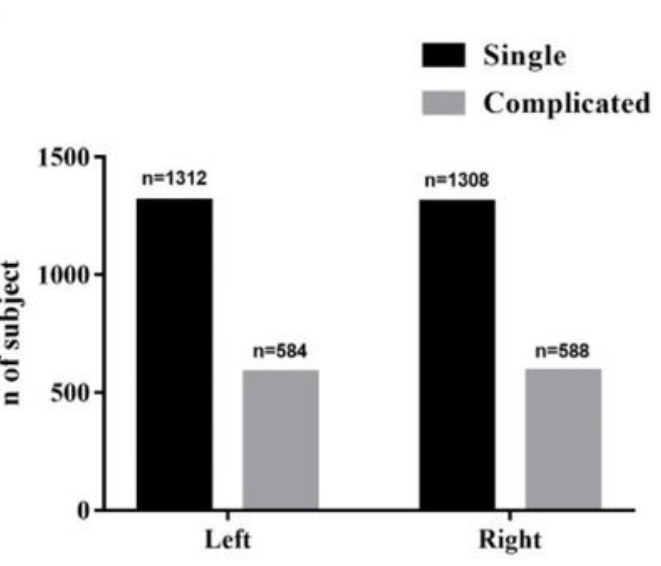

Figure 3

Characteristics of the study population examined teeth by genders, age groups, and sides. a The distribution of MFMs with DLRs between male and female. $b$ The distribution complicated root canal configurations of MLIs between male and female. c The distribution of MFMs with DLRs between 
different age groups. $d$ The distribution complicated root canal configurations of MLIs between different age groups. e The distribution of MFMs with DLRs between sides. $f$ The distribution complicated root canal configurations of MLIs between sides. 